\title{
Regulation of Aromatic Metabolism in Fungi: Selection of Mutants of the Yeast Rhodotorula mucilaginosa with Nystatin
}

\author{
By K. A. COOK \\ Biological Laboratories, University of Kent, Canterbury $\mathrm{CT}_{2}{ }_{7} \mathrm{NJ}$, Kent
}

(Received 6 May I974; revised 20 June 1974)

SUMMARY

The antifungal antibiotic nystatin has been successfully used to select mutants of the yeast Rhodotorula mucilaginosa from populations consisting largely of wildtype organisms. The most efficient selection technique has been found by a study of the duration of the nitrogen starvation period, the composition of the starvation medium, and the concentration of nystatin. Several mutants have been obtained which are unable to utilize either or both of the aromatic carbon sources $p$-hydroxybenzoate and protocatechuate.

\section{INTRODUCTION}

The search for a technique of auxotrophic selection in fungi, similar to the penicillin method widely used in bacteria (Davis, I948; Lederberg \& Zinder, I948), led to the discovery that the polyene antibiotic nystatin produced by Streptomyces noursei (Hazen \& Brown, I95I; Brown \& Hazen 1957), was effective in selecting auxotrophs of some fungi where these were present in mixed populations with prototrophs. Its selective action has been demonstrated with Saccharomyces cerevisiae (Moat, Peters \& Srb, 1959; Snow, 1966; Thouvenot \& Bourgeois, 1971), Penicillium chrysogenum (MacDonald, 1968) and Aspergillus nidulans (Ditchburn \& MacDonald, I97I). Nystatin had not previously been used to select mutant strains of fungi with defects in catabolic pathways.

This paper describes the use of nystatin in the selection of $N$-methyl- $N^{\prime}$-nitro- $N$-nitrosoguanidine (NTG)-induced mutants of the yeast Rhodotorula mucilaginosa blocked in the catabolism of $p$-hydroxybenzoic acid. The physiological conditions giving the optimum enrichment of mutant over wild-type organisms were determined and the final selection procedure used in the isolation of mutants unable to grow at the expense of $p$-hydroxybenzoate. This substrate is utilized as a carbon and energy source by the wild-type strain, and metabolized by the following route (Cain, Bilton \& Darrah, 1968):

$p$-Hydroxybenzoate $\longrightarrow$ protocatechuate $\longrightarrow 3$-carboxymuconate

$\longrightarrow$ 3-carboxymuconolactone $\longrightarrow$ 3-oxoadipate $\rightarrow$ succinate + acetate.

\section{METHODS}

Organisms. Wild-type Rhodotorula mucilaginosa was isolated as an aerial contaminant on $p$-hydroxybenzoate medium (Cain et al. 1968) and identified by the Brewing Industry Research Foundation, Nutfield, Surrey.

Strain PHBI, a mutant which lacked the enzyme $p$-hydroxybenzoate hydroxylase [4hydroxybenzoate, $\mathrm{NAD}(\mathrm{P}) \mathrm{H}$ : oxygen oxidoreductase (3-hydroxylating)] (EC. I. I4. I3.I), 
was isolated by the author after NTG treatment of the wild-type strain but without the subsequent imposition of a selection procedure, and was used as a control organism in the development of the nystatin selection procedure described below.

Media. All media were prepared in a mineral salts base of the following composition (g/l water): $\left(\mathrm{NH}_{4}\right)_{2} \mathrm{SO}_{4}, 0.5 ; \mathrm{KH}_{2} \mathrm{PO}_{4}, \mathrm{I} \cdot 0 ; \mathrm{MgSO}_{4} \cdot 7 \mathrm{H}_{2} \mathrm{O}, 0.05 ;$ Bacto yeast extract (Difco), $0 \cdot \mathrm{I}$; trace elements solution (Barnet \& Ingram, 1955 ), $\mathrm{I} \cdot 0 \mathrm{ml}$. The $\mathrm{pH}$ was adjusted to 5.5 (unless otherwise stated) by the addition of concentrated ortho-phosphoric acid and the medium sterilized by autoclaving. Carbon sources were prepared as neutral stock solutions, sterilized separately by autoclaving (glucose and sodium $p$-hydroxybenzoate) or filtration (sodium protocatechuate $-3,4$-dihydroxybenzoate), and added aseptically to the mineral salts base.

Complete medium (CM) contained Io mM-glucose as carbon source while minimal medium (MM) contained ro mM- $p$-hydroxybenzoate. Nitrogen-free medium ( $\mathrm{N}$-free) comprised mineral salts base from which $\left(\mathrm{NH}_{4}\right)_{2} \mathrm{SO}_{4}$ and yeast extract were omitted and contained either Io mM-glucose or $10 \mathrm{~mm}$-p-hydroxybenzoate as carbon sources, as indicated in the text.

The appropriate liquid media were solidified by the addition of $I \cdot 5 \%(\mathrm{~W} / \mathrm{v})$ purified agar (Oxoid).

Growth and maintenance of organisms. All strains were maintained by monthly subculture on $\mathrm{CM}$ slopes at $30^{\circ} \mathrm{C}$ and stored at $4{ }^{\circ} \mathrm{C}$.

Liquid cultures $\left(40 \mathrm{ml}\right.$ of medium) were grown at $30^{\circ} \mathrm{C}$ in flasks $(250 \mathrm{ml})$ with side-arms on a rotary shaker (Mk 3, L.H. Engineering Co., Stoke Poges, Buckinghamshire) operated at $\mathrm{I} 40 \mathrm{rev} . / \mathrm{min}$.

Enumeration of organisms. Cell density was estimated turbidimetrically with an EEL colorimeter (Evans Electroselenium Ltd, Halstead, Essex) and related to cell numbers obtained by direct counting by use of a calibration curve. A linear correlation was obtained with suspensions containing up to $6 \times 10^{7}$ organisms $/ \mathrm{ml}$.

Viable counts were performed in quadruplicate on solid media by the spread plate method, with all dilutions made in saline $(0.85 \%, \mathrm{w} / \mathrm{v}, \mathrm{NaCl}$ solution).

Mutagenesis. A wild-type culture growing exponentially on CM was treated with NTG under experimentally determined optimal conditions $\left(50 \mu \mathrm{g} / \mathrm{ml}\right.$ of culture at $30^{\circ} \mathrm{C}$ and $\mathrm{pH} 5.5$ for $30 \mathrm{~min}$ ). After this time organisms were harvested by centrifugation, washed three times in sterile potassium phosphate buffer $(0.025 \mathrm{M}, \mathrm{pH} \mathrm{6.5)}$, and subcultured on to fresh $\mathrm{CM}$ to allow segregation of mutant and non-mutant nuclei from multinucleate cells and permit the phenotypic expression of any induced mutations (Witkin, 1956).

Under these conditions approximately $5 \%$ of the organisms survived NTG treatment.

Isolation and characterization of mutants. Organisms which survived NTG treatment and subsequent selection were plated on to $\mathrm{MM}$ and incubated at $30^{\circ} \mathrm{C}$ for $48 \mathrm{~h}$. Under these conditions organisms unable to utilize $p$-hydroxybenzoate formed microcolonies (see below). Microcolonies thus isolated were patched on to $\mathrm{CM}$ and subsequently tested for their appropriate nutritional requirements by the replication technique of Lederberg \& Lederberg (1952). Putative mutants were then streaked several times on CM and single colonies isolated which were streaked on to $\mathrm{CM}$ and stored at $4{ }^{\circ} \mathrm{C}$.

The site of the enzymic lesion in mutants which had lost the ability to grow on one or more aromatic carbon sources was determined by enzyme analysis of extracts of the mutants after growth at the expense of $10 \mathrm{mM}$-glucose in the presence of $20 \mathrm{~mm}$ - $p$-hydroxybenzoate. Preparation of cell-free extracts and measurement of the relevant enzymes are described in detail in the following paper (Cook \& Cain, 1974). 


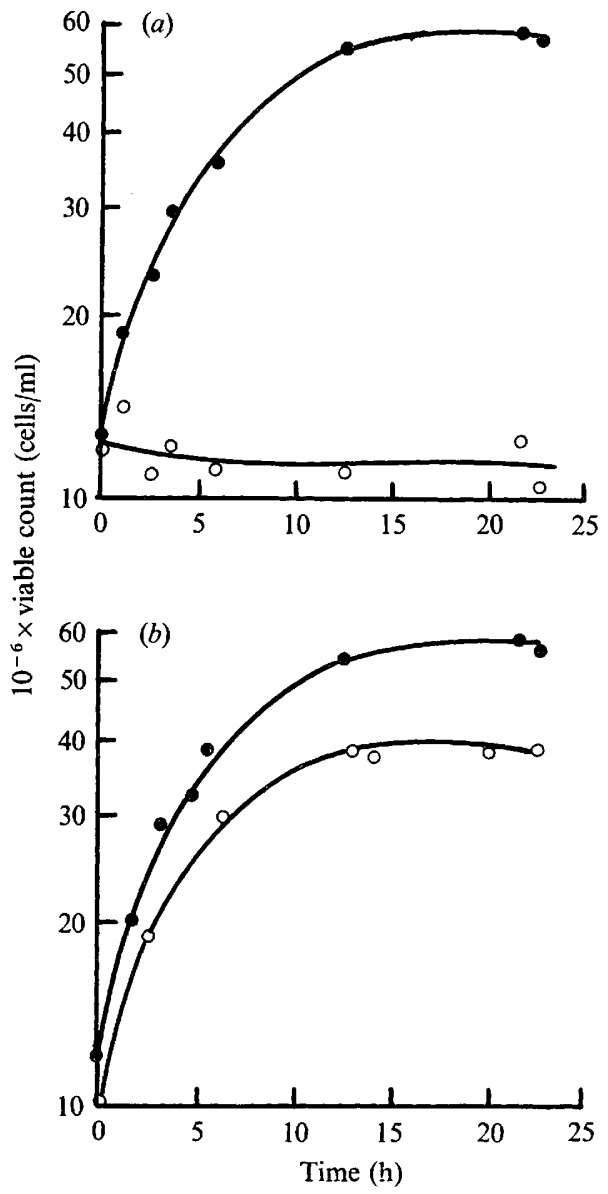

Fig. I. Nitrogen starvation of $R$. mucilaginosa in the presence of different carbon sources. Strain PHBI $(a)$ and the wild-type strain $(b)$ were incubated in $\mathrm{N}$-free media containing Io mm-glucose $(\odot)$ and $10 \mathrm{~mm}-p$-hydroxybenzoate $(O)$ as carbon sources.

Preparation of nystatin. Nystatin (Nystan, E. R. Squibb \& Sons, Liverpool) was prepared as a suspension in water at $2 \mathrm{mg} / \mathrm{ml}$ and the $\mathrm{pH}$ adjusted to 8.5 with $5 \mathrm{M}-\mathrm{NaOH}$. The clear yellow solution obtained was sterilized by filtration through a Millipore membrane $(0.22 \mu \mathrm{m}$ pore size, $0.47 \mathrm{~mm}$ thick) and used immediately because of its limited stability (Dutcher, Walters \& Wintersteiner, 1955). One $\mathrm{mg}$ of nystatin was equivalent to 4950 units.

\section{RESULTS}

Effect of starvation conditions on the wild type and on strain $\mathrm{PHBI}$

The effectiveness of nystatin in selecting fungal auxotrophs, when these are present in mixed populations with prototrophs, rests upon its differential effect upon growing and non-growing organisms (Snow, I966; Ditchburn \& MacDonald, I97I; Thouvenot \& Bourgeois, 1971). A period of starvation must therefore be imposed before nystatin treatment to ensure that mutant organisms have exhausted their pool of endogenous metabolites and will be unable to grow when transferred to MM. The duration of the starvation period 


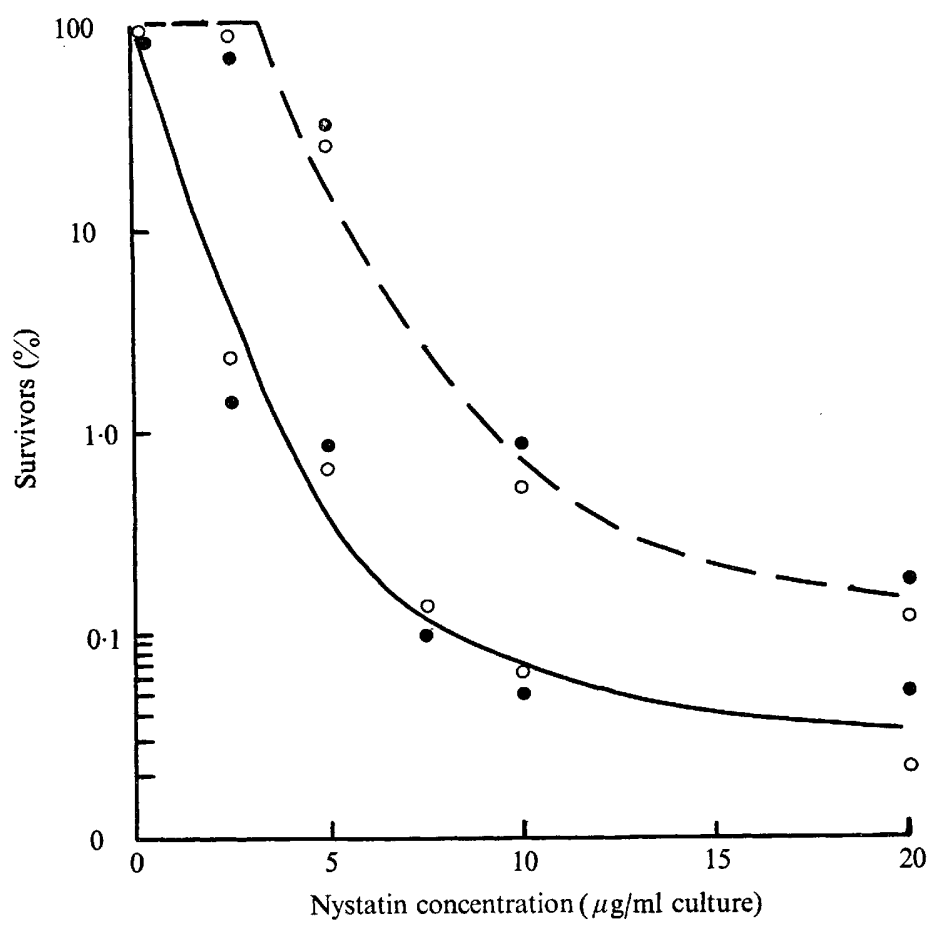

Fig. 2. Differential effects of nystatin on $R$. mucilaginosa. The wild-type strain $(-)$ and mutant strain PHBI (---) were nitrogen starved in the presence of $10 \mathrm{~mm}$-glucose ( $)$ and $10 \mathrm{~mm}-p-$ hydroxybenzoate $(O)$ before exposure to the indicated concentrations of nystatin. The number of surviving organisms was estimated from viable counts on CM.

required for $R$. mucilaginosa was determined as follows: organisms from an exponential culture of strain PHBI on CM were harvested by centrifugation, washed three times with physiological saline, and transferred to $\mathrm{N}$-free medium containing either Io mM-glucose or ro mM-p-hydroxybenzoate as carbon sources. Cultures were incubated at $30^{\circ} \mathrm{C}$ and growth monitored turbidimetrically for $24 \mathrm{~h}$ (Fig. I $a$ ). With glucose as carbon source cell numbers increased approximately fourfold within I3 h (cf. Thouvenot \& Bourgeois, I97I, using $S$. cerevisiae). When glucose was replaced by $p$-hydroxybenzoate, now exposing the mutant strain to conditions of both nitrogen and carbon starvation, no increase in cell numbers was observed. These data suggested that strain PHBI was incapable of multiplication in the absence of a utilizable carbon source; however, no loss of viability was observed during incubation under these conditions, viable cell counts of $\mathrm{I} \cdot \mathrm{I} \times 10^{7}, \mathrm{I} \cdot 2 \times 10^{7}, \mathrm{I} \cdot 3 \times 10^{7}$ and $\mathrm{I} \cdot 2 \times 1 \mathrm{O}^{7}$ being obtained after $2 \cdot 5,6, \mathrm{I} 5$ and $23 \mathrm{~h}$ starvation respectively.

Rhodotorula mucilaginosa wild-type was capable of metabolizing both glucose and $p$ hydroxybenzoate and hence similar curves were obtained in $\mathrm{N}$-free media containing either carbon source (Fig. I $b$ ), and cell numbers increased between three- and fourfold within $\mathrm{I} 2 \mathrm{~h}$.

Differential effects of nystatin on pure cultures of the wild type and of strain $\mathrm{PHB}$

The maximum lethal effect of nystatin is dependent on its efficient absorption by prototrophs, which occurs at $30{ }^{\circ} \mathrm{C}$ and $\mathrm{pH} 4.2$ in the presence of a utilizable carbon source (Lampen, Morgan, Slocum \& Arnow, 1959). Similarly nystatin exerts a maximal effect on 
well-aerated cultures in exponential phase (Harman \& Masterson, 1957; Moat, Barnes \& McCurley, I966).

Organisms from an exponential culture of the wild-type strain on CM were transferred to $\mathrm{N}$-free medium containing either glucose or $p$-hydroxybenzoate as carbon source and incubated at $30^{\circ} \mathrm{C}$ for $20 \mathrm{~h}$, as described above. After starvation, organisms were harvested, washed and inoculated into each of a series of flasks of $\mathrm{MM}$ adjusted to $\mathrm{pH} 4 \cdot 2$ at a final density of $5 \times 10^{6}$ organisms $/ \mathrm{ml}$. When the cultures had reached exponential phase (after approximately Io h) varying amounts of nystatin were added to the different cultures to give a series of concentrations of between 2.5 and $20 \mu \mathrm{g} / \mathrm{ml}$ of culture, and the cultures incubated at $30^{\circ} \mathrm{C}$ in the dark. Samples were removed I $\mathrm{h}$ later, immediately diluted Ioo-fold in physiological saline to dilute the residual nystatin to a sublethal concentration, and viable counts performed.

The mutant strain, PHBI, was treated in an identical manner to that described above for the wild type, imposing a further starvation period upon it as it was unable to grow on MM. However, no loss of viability was observed during this additional starvation period, irrespective of the carbon source previously utilized in the $\mathrm{N}$-free medium, viable cell counts of $\mathrm{I} \cdot 9 \times 10^{6}, 2 \cdot 0 \times 10^{6}, \mathrm{I} \cdot 8 \times 10^{6}$ and $\mathrm{I} \cdot 9 \times 10^{6}$ being obtained after $3,6,9$ and $\mathrm{Io} h$ incubation respectively.

It is evident (Fig. 2) that under the experimental conditions described strain PHBI was considerably more resistant to nystatin than was the wild-type strain. At nystatin concentrations of up to $4 \mu \mathrm{g} / \mathrm{ml}$ of culture the viability of strain PHBI was unaffected while at the same concentration $99 \%$ of the wild-type organisms were killed. The nature of the carbon source utilized in the starvation medium had no effect on the subsequent survival of either strain after nystatin treatment and a starvation medium containing Io mM-glucose as carbon source was adopted for subsequent use.

\section{Effects of nystatin on a mixed population of the wild type and strain $\mathrm{PHB} \mathrm{I}$}

The differential effects of nystatin on the wild type and on strain PHBI (Fig. 2) suggested the suitability of the antibiotic for use as a selective agent provided that the response of the two strains together in a mixed culture did not differ significantly from their individual responses to the antibiotic. This was investigated by mixing nitrogen-starved cultures of the two strains before nystatin treatment. Snow (1966) found that to avoid syntrophy between prototrophic and auxotrophic stains of $S$. cerevisiae (and hence subsequent growth and destruction of the auxotrophs by nystatin) it was advisable not to exceed a cell density of $10^{7}$ organisms/ml during nystatin selection. Hence after nitrogen starvation wild type and mutant cultures of $R$. mucilaginosa were inoculated in equal numbers into MM to give a combined initial density of $5 \times 10^{6}$ organisms $/ \mathrm{ml}$; nystatin was added after approximately $9 \mathrm{~h}$, when a cell density of $\mathrm{I} \times 10^{7}$ organisms $/ \mathrm{ml}$ had been attained. After exposure to nystatin for $\mathrm{I} h$ organisms were plated on to both $\mathrm{MM}$ and $\mathrm{CM}$ and incubated at $30^{\circ} \mathrm{C}$. Discrimination between surviving wild-type and mutant organisms was facilitated by the fact that mutants formed microcolonies on MM. When successive samples from a mixed culture in liquid MM were plated on to $\mathrm{CM}$ and $\mathrm{MM}$ the number of mutant organisms, estimated from the difference in normal colony counts on CM and MM, was in close agreement with the number of microcolonies on MM. Hence in subsequent experiments the number of mutant organisms surviving nystatin treatment was estimated from the number of microcolonies appearing on MM.

From the data obtained using individual pure cultures of the wild-type and strain PHBI treated with nystatin (Fig. 2) it was possible to calculate the theoretical enrichment of mutant 


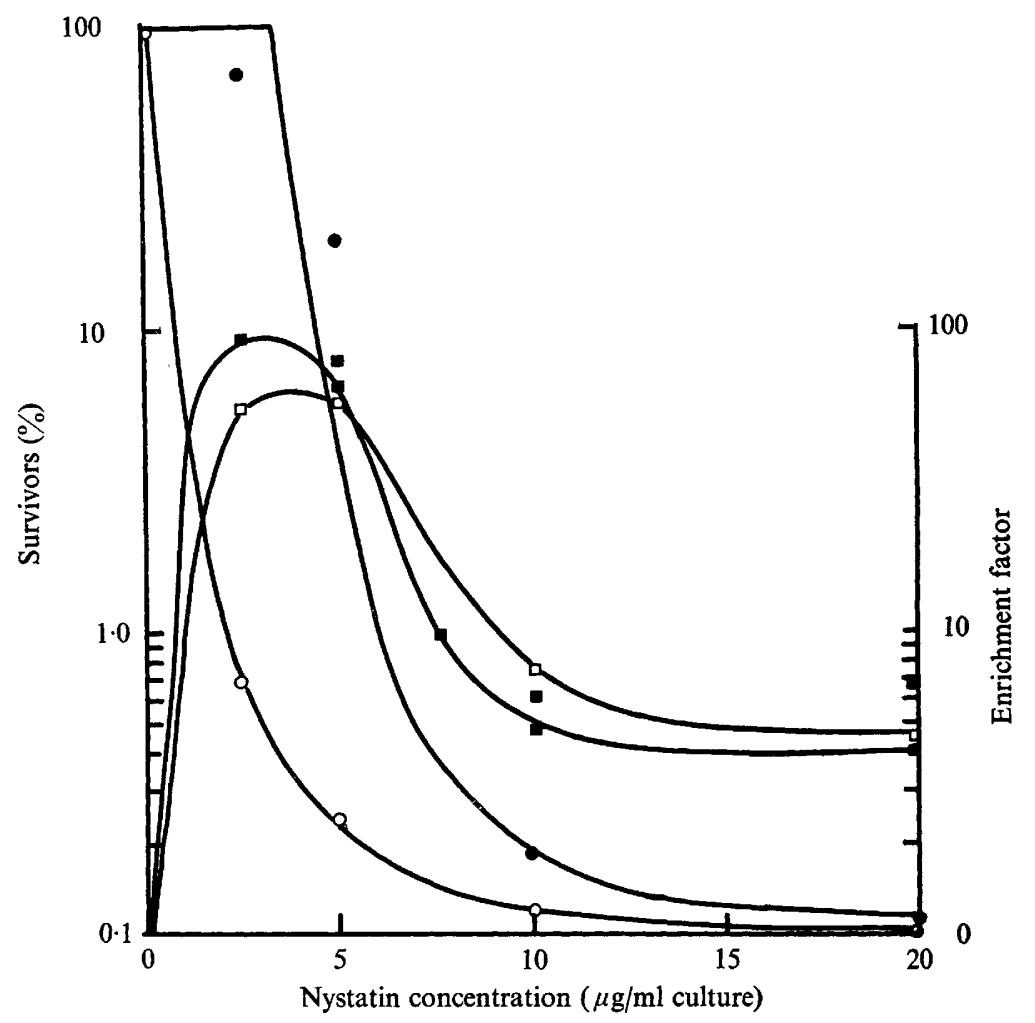

Fig. 3. Effects of nystatin on a mixed population of $R$. mucilaginosa wild type and strain PHBI. The survival of the wild-type strain $(O)$ and strain PHBI (O) was determined in the presence of nystatin at the concentrations indicated, and the theoretical enrichment ( $\square$ ) compared with the experimentally determined enrichment $(\square)$ of strain PHBI over the wild-type strain deduced from the results. The enrichment factor $\left(E_{F}\right)$ is defined in the text.

over wild-type organisms expected in a mixed culture experiment, assuming that their susceptibilities to nystatin remained unaltered under these conditions. Comparison of this theoretical enrichment data with data obtained using an actual mixed culture should then indicate whether the behaviour of a mixed culture differed from that of its individual components towards nystatin. The enrichment factor, $E_{F}$, was defined as:

$$
E_{F}=\frac{\text { ratio of mutant to wild-type organisms after nystatin treatment }}{\text { ratio of mutant to wild-type organisms before nystatin treatment }} .
$$

The data presented in Fig. 3 indicated that the behaviour of a mixed culture towards nystatin did not vary significantly from the behaviour of individual pure cultures under the conditions used, and that approximately I0o-fold enrichment of mutant over wild-type organisms was possible using the selection procedure described here.

\section{Production and selection of mutants}

From the results described above, the following standard procedure was adopted: after mutagenesis of a culture of the wild-type strain and subsequent growth of the survivors to exponential phase on CM, organisms were harvested by centrifugation, washed with physiological saline, and inoculated into $\mathrm{N}$-free medium with $10 \mathrm{~mm}$-glucose as carbon 


\section{Table I. Growth characteristics of mutants obtained after nystatin selection of NTG-treated wild-type R. mucilaginosa}

Survivors from nystatin selection were plated on to $\mathrm{MM}$ and incubated at $30{ }^{\circ} \mathrm{C}$ for $48 \mathrm{~h}$, after which time mutants formed micro-colonies. Micro-colonies were patched on to $\mathrm{CM}$ and subsequently replicated on to different media; + and - indicate growth and no growth respectively.

$\begin{array}{ccccc}\text { Group } & \text { No. of mutants } \\ \text { isolated } & \overbrace{\text { Glucose }}^{\text {Growth of mutants, on carbon sources }} \\ \text { I } & 2 & + & p \text {-Hydroxybenzoate } & \text { Protocatechuate } \\ 2 & 2 \mathrm{I} & + & + & - \\ 3 & 26 & + & - & +\end{array}$

source. After $20 \mathrm{~h}$, organisms were transferred to $\mathrm{MM}$ (adjusted to $\mathrm{pH} 4 \cdot 2 ; 5 \times \mathrm{IO}^{6}$ organisms $/ \mathrm{ml}$ of culture) and grown for $\mathrm{I} 0 \mathrm{~h}$, by which time the cell density had reached approximately $10^{7}$ organisms $/ \mathrm{ml}$. At this point nystatin was added (final concentration of $3.75 \mu \mathrm{g} / \mathrm{ml}$ of culture) and the culture incubated at $30^{\circ} \mathrm{C}$ in the dark for I h. Culture samples were then diluted Ioo-fold and survivors were plated on to MM and allowed to grow for $48 \mathrm{~h}$, when mutant organisms formed micro-colonies. Mutants were subsequently characterized as described in Methods.

In the course of two typical experiments 49 stable mutants were obtained (Table I), although it was possible that several of the mutants having similar growth characteristics and obtained in the course of the same manipulations were derived from the same clone. Three distinct groups of mutants were obtained and from their growth characteristics it appears that group I mutants were unable to take up protocatechuate from the medium, that group 2 mutants either lacked $p$-hydroxybenzoate hydroxylase or were impermeable to $p$-hydroxybenzoate in the medium, and that group 3 mutants were deficient in some enzyme involved in the subsequent degradation of protocatechuate or were impermeable to both $p$-hydroxybenzoate and protocatechuate in the medium.

The enzymic analysis of representative mutants from groups 2 and 3 is described in Cook \& Cain (1974).

\section{DISCUSSION}

The nystatin selection procedure described here is a modification of a procedure for the selection of $S$. cerevisiae mutants proposed by Snow (I966) and subsequently refined by Thouvenot \& Bourgeois (1971). However, in addition to the modification of the technique for use with $R$. mucilaginosa it has been adapted to the selection of mutants deficient in the catabolism of aromatic carbon compounds, whereas previous workers have concentrated on the selection of mutants defective in the biosynthesis of nitrogenous compounds. The magnitude of the enrichment of $R$. mucilaginosa mutants over the wild-type strain was approximately sevenfold less than that reported for $S$. cerevisiae mutants (Thouvenot \& Bourgeois, 1971). A likely reason for this is the greater sensitivity of $R$. mucilaginosa towards nystatin; exposure of wild-type $R$. mucilaginosa to $20 \mu \mathrm{g}$ nystatin $/ \mathrm{ml}$ (I00 units $/ \mathrm{ml}$ ) resulted in the survival of $0.035 \%$ ' of the organisms compared with a survival of $0.4 \%$ with $S$. cerevisiae. The different susceptibility of mutant strains of the two organisms were even more pronounced, only $0.15 \%$ of cells of PHB I surviving treatment with $20 \mu \mathrm{g}$ antibiotic $/ \mathrm{ml}$, whereas $85 \%$ of a mutant strain of $S$. cerevisiae survived under similar conditions. These factors necessitated the use of a considerably lower concentration of nystatin during selection of $R$. mucilaginosa mutants. 
K. A. COOK

The mutants produced during the course of this study, namely those lacking the enzymes $p$-hydroxybenzoate hydroxylase and protocatechuate 3,4-dioxygenase, have subsequently been utilized in the elucidation of the specific inducers operative in the metabolic control of p-hydroxybenzoate degradation (Cook \& Cain, I974).

This work was supported by grant $\mathrm{B} / \mathrm{RG} / 3509$ from the Science Research Council to Dr R. B. Cain. I thank Dr Jane North and Dr Cain for their advice and for reading this manuscript, and Susan Black for her able technical assistance.

\section{REFERENCES}

Barnet, J. A. \& INGRAM, M. (1955). Techniques in the study of yeast assimilation reactions. Journal of Applied Bacteriology 18, I3I-I 37.

Brown, R. \& HAZEN, E. L. (1957). Present knowledge of nystatin, an antifungal antibiotic. Transactions of the New York Academy of Sciences 19, 447-456.

Cain, R. B., Bilton, R. F. \& Darrah, J. A. (1968). The metabolism of aromatic acids by micro-organisms. Metabolic pathways in the fungi. Biochemical Journal 108, 797-828.

СоoK, K. A. \& CAIN, R. B. (1974). Regulation of aromatic metabolism in the fungi: metabolic control of the 3-oxoadipate pathway in the yeast Rhodotorula mucilaginosa. Journal of General Microbiology 85, $37-50$.

DAVIS, B. D. (1948). Isolation of biochemically deficient mutants of bacteria with penicillin. Journal of the American Chemical Society $70,4267$.

Ditchiurn, P. \& MACDonald, K. D. (1971). The differential effects of nystatin on growth of auxotrophic and prototrophic strains of Aspergillus nidulans. Journal of General Microbiology 67, 299-306.

Dutcher, J. D., Walters, D. R. \& Wintersteiner, O. D. (1955). Studies on the chemical and physical properties and structure of nystatin. In Therapy of Fungus Diseases. An International Symposium, pp. 168-1 76. Edited by T. H. Sternberg and M. D. Newcomer. Boston, Massachusetts: Little, Brown \& Co.

Harman, J. W. \& Masterson, J. G. (1957). The mechanism of nystatin action. Irish Journal of Medical Science 378, 249-253.

Hazen, E. L. \& Brown, R. (I95I). Fungicidin, an antibiotic produced by a soil actinomycete. Proceedings of the Society for Experimental Biology and Medicine 76, 93-97.

Lampen, J. O., Morgan, E. R., Slocum, A. \& Arnow, P. (1959). Absorption of nystatin by microorganisms. Journal of Bacteriology 78, 282-289.

LeDerberG, J. \& LeDERBeRG, E. M. (I952). Replica plating and indirect selection of bacterial mutants. Journal of Bacteriology 63, 399-406.

LeDERBERG, J. \& ZiNDER, N. (1948). Concentration of biochemical mutants of bacteria with penicillin. Journal of the American Chemical Society 7o, 4267-4268.

MacDonald, K. D. (1968). The selection of auxotrophs of Penicillium chrysogenum with nystatin. Genetical Research II, 327-330.

MoAT, A. G., BARNes, I. J. \& MCCuRLey, E. (I966). Factors affecting the survival of auxotrophs and prototrophs of Saccharomyces cerevisiae in mixed populations. Journal of Bacteriology 92, 297-301.

MoAt, A. G., Peters, N. \& SRB, A. M. (1959). Selection and isolation of auxotrophic yeast mutants with the aid of antibiotics. Journal of Bacteriology 77, 673-684.

SNOw, R. (1966). An enrichment method for auxotrophic yeast mutants using the antibiotic nystatin. Nature, London, 211, 206-207.

Thouvenot, D. R. \& Bourgeors, C. M. (197I). Optimisation de la sélection de mutants de Saccharomyces cerevisiae par la nystatine. Annales de l'Institute Pasteur 120, 617-625.

WITKIN, E. M. (1956). Time, temperature and protein synthesis: a study of uitra-violet induced mutations in bacteria. Cold Spring Harbor Symposia on Quantitative Biology 21, I23-136. 